\title{
Geomorphological of hazard maps in ABAH Urban, KSA
}

\author{
Mena Elassal ${ }^{1}$
}

\begin{abstract}
:
Abha Urban is located in Asir region, southwest of Saudi Arabia. Geomorphology is one of the most influential controls in urban planning, which gives an idea about the variations in landscape/landforms and indirectly facilitates in evaluating the resources of an area. for detailed study, multi-temporal satellite remote sensing imageries and ArcGIS were used to compile and create database that provided a visual and historical perspective of the urban expansion practiced in the study area since 1970 to 2018. the different thematic map such as land use change pattern analysis, topography, and geomorphology highlight the profound changes to the landscape/landform that have incrementally developed over time. Through studying geological structures, analyzing the height and slope of the surface - topography of the surface - and their impact on the urban expansion of the region, this research illustrates how geomorphological expert knowledge can be integrated in a GIS and RS for the evaluation of Geomorphological hazards.

Geomophological hazard assessment is an important component of natural hazard risk assessment. This paper presents GIS-based geomorphological hazard mapping in Abha urban, this includes the integration of published geological, geomorphological, and other data into GIS, and generation of new map products, combining governmental concerns and legal restrictions. Detailed geomorphological hazard maps for flooding zones and earth movement potential, further the paper illustrates the application of vulnerability maps dealing with the effect of hazard on urban areas, these maps can help to initiate appropriate measures to mitigate the probable hazards in the area. And trying to propose appropriate solutions for urban expansion in the light of the Geomorphological hazard's studies. [Bul. Soc. Géog. d’Égypte, 2019, 92: 53-75].
\end{abstract}

KEYWORD: Urban Geomorphology; geomorphic hazards; GIS; RS

\section{Introduction:}

Geomorphological information and geomorphological approach are integrated in hazard zonation. It's research presents digital thematic maps prepared on the basis of a detailed geomorphological map stored in a GIS and field work. Geomorphological hazards are well known worldwide (Lazzari et al, 2006) indicated that the integrated analysis between territorial data (geology, geomorphology and climate) and historical documents showed that the geomorphological hazard has been accentuated by the intense human activity. It's work also produced evidence that the human interventions occurring during the historical period have been a determining factor in increasing the hazard level and accelerating the preexisting morphological processes. There are different elements dealing with geomorphological phenomena including hazard, vulnerability, and risk. (Crichton, 1999) defined the risk as the probability of a loss, and this depends on three elements "hazard, vulnerability and exposure". If any of these three elements increases or decreases, then risk in that area increases or decreases, respectively. Hazard refers to a characteristic of or phenomenon from the natural environment which has the potential for causing damage to society. (AlcantaraAyala,2002) defined the term hazard as it is often associated with different agents or processes. Some of these parameters include atmospheric, hydrologic, geologic, biologic and technologic. As such natural hazards are threatening events, capable of producing damage to the physical and social space in which they take place not only at the moment of their

1- Assistant Professor, Geomorphology and Geoarchaeology, Faculty of Humanities, King Khalid University, KSA. 
occurrence, but in the long-term, due to their associated consequences. Natural disasters occur all over the world; however, their impact in developing countries is greater due to the geographical location in zones highly susceptible to natural hazards (natural vulnerability), and also due to the different types of economic, social, political and cultural vulnerabilities that exist (Alcantara-Ayala, 2002). The current research deals with the evaluation of the geomorphological hazards (flash floods, mass movements, and Seismic) to delimitate the most susceptibility areas for flash floods and mass movements in Abha urban.

\section{Study Area}

Abha located in the Emirate of Asir in the southwestern part of the Kingdom of Saudi Arabia. Located between $\square 17 \square 57 \square 59$ to $\square 18 \square 37 \square 18 \mathrm{~N}$ latitude, and between $\square 42 \square 16$ $\square 39$ to $\square 43 \square 02 \quad \square 38$ E longitude (Fig.1). The study area is located on the heights of the Sarawat, which is part of the highest regions in the Kingdom of Saudi Arabia, and the height ranges from 1900 to $3000 \mathrm{~m}$ above sea level, and increases the height to $3000 \mathrm{~m}$, as is the mountain of As Souda near the city of Abha. The study area is district represents one of the main drainage basin systems in KSA, the district is subjected to flash floods annually, in recent years the frequency of flash floods had increased and caused significant damages to the infrastructure and other facilities in the area such as Bisha, Rania and Wadi Abha.



Fig: (1) The location of study area.

\section{Data Used and Methodology}

The study was an analysis of topographic maps of different scale, Geological maps, Aerial photos, and various satellite images (Landsat- World view 2- Ikonos, Quick Bird), and Google earth. By using Arc GIS Desk Top 10.3. and field study. 


\section{Results and Discussion}

\section{Geology}

The study area is a part of the Arabian Shield, which consists of a series of ancient volcanic rocks, dating back to 800-1000 million years. Which are composed of the sedimentary, volcanic, and metamorphic rocks formed during the protozoic periods? These rocks have been exposed since their formation and their origin to the movement of many distortion and transformation, which caused the disappearance of most of their original properties and cut with many massive to layered plutons, sills, dikes and irregular bodies. and different directions and shapes (walliam, 1983), as in the geological map scale of 1: 250,000:

Most of these rocks originated in an environment Islands, it consists of the following: Halaban, Ablah, Jiddah, Bahah, Baish groups. (Fig.2), and Table (1) shows the geological formations and area $\left(\mathrm{km}^{2}\right)$ and the description Formation groups.



Fig (2): Geological Map of study area. 
Table: (1) The Geological formation groups and area $\left(\mathrm{km}^{2}\right)$.

\begin{tabular}{|c|c|c|c|}
\hline Formation & Formation group & Area $(\mathrm{km} 2)$ & $\%$ \\
\hline Bt & JIDDAH GROUP & 454.9367668 & 12.54394588 \\
\hline Dg & GRANITE SUITE & 36.26797643 & 1.000014874 \\
\hline di3 & $\begin{array}{c}\text { DIORITIC AND } \\
\text { GABROIC ROCKS }\end{array}$ & 35.42237577 & 0.976699175 \\
\hline Gb & $\begin{array}{c}\text { Dioritic and gabbric } \\
\text { rocks }\end{array}$ & 16.77566293 & 0.46255441 \\
\hline Gdh & GRANITE SUITE & $\mathbf{3 6 . 8 3 7 9 5 7 0 3}$ & 1.015730917 \\
\hline Gdm & $\begin{array}{l}\text { GRANODIORITE AND } \\
\text { GRANITE SUITE }\end{array}$ & 9.761021442 & 0.269140095 \\
\hline Gdn & $\begin{array}{l}\text { GRANODIORITE AND } \\
\text { GRANITE SUITE }\end{array}$ & 716.7615376 & 19.76322556 \\
\hline Gdv & $\begin{array}{l}\text { GRANODIORITE AND } \\
\text { GRANITE SUITE }\end{array}$ & 129.0481559 & $\mathbf{3 . 5 5 8 2 3 7 5 4 4}$ \\
\hline Ghn & $\begin{array}{l}\text { GRANODIORITE AND } \\
\text { GRANITE SUITE }\end{array}$ & 341.3841204 & 9.412965151 \\
\hline Grb & GRANITE SUITE & 489.7767206 & 13.50458597 \\
\hline Hkv & $\begin{array}{l}\text { HALABAN GROUP } \\
\text { WITHIN THE } \\
\text { KHADRA BELT }\end{array}$ & 55.97201358 & 1.543313182 \\
\hline Jba & $\begin{array}{l}\text { JIDDAH AND BAHAH } \\
\text { GROUPS, UNDIVIDED }\end{array}$ & 426.9820544 & 11.77315217 \\
\hline Jbg & $\begin{array}{l}\text { JIDDAH AND BAHAH } \\
\text { GROUPS, UNDIVIDED }\end{array}$ & 179.620512 & 4.952666275 \\
\hline $\mathbf{J t}$ & JIDDAH GROUP & 262.8534491 & 7.247643368 \\
\hline $\mathbf{M g}$ & $\begin{array}{c}\text { DIORITIC AND } \\
\text { GABROIC ROCKS }\end{array}$ & 9.429765029 & 0.260006381 \\
\hline Mgy & $\begin{array}{c}\text { DIORITIC AND } \\
\text { GABROIC ROCKS }\end{array}$ & 35.26781114 & 0.972437373 \\
\hline oEw & $\begin{array}{c}\text { SEDIMENTARY, } \\
\text { VOLCANIC, AND } \\
\text { METAMORPHIC } \\
\text { ROCKS }\end{array}$ & 70.0294229 & 1.930917339 \\
\hline Qal & $\begin{array}{c}\text { SEDIMENTARY, } \\
\text { VOLCANIC, AND } \\
\text { METAMORPHIC } \\
\text { ROCKS } \\
\end{array}$ & 63.26653672 & 1.744444659 \\
\hline Qdn & TONALITE SUITE & 200.4152484 & 5.526038372 \\
\hline Tb & $\begin{array}{c}\text { SEDIMENTARY, } \\
\text { VOLCANIC, AND } \\
\text { METAMORPHIC } \\
\text { ROCKS }\end{array}$ & 10.03395118 & 0.276665572 \\
\hline Tgm & $\begin{array}{l}\text { GRANODIORITE AND } \\
\text { GRANITE SUITE }\end{array}$ & 33.10384432 & 0.912770438 \\
\hline Thb & GRANITE SUITE & 2.751189671 & 0.075858398 \\
\hline Tl & $\begin{array}{c}\text { SEDIMENTARY, } \\
\text { VOLCANIC, AND } \\
\text { METAMORPHIC } \\
\text { ROCKS }\end{array}$ & 8.505197632 & 0.234513336 \\
\hline tn & TONALITE SUITE & 1.540406968363 & 0.042473555 \\
\hline Total & - & 3626.743698 & 100 \\
\hline
\end{tabular}




\section{Topographic characteristics:}

The area of the study is characterized by its elevation from the sea surface. It is located in the Sarra Asir mountain range, which is the most complex and morphological region in the Kingdom of Saudi Arabia. It shows the mountains with high peaks and it descends strongly towards the sea. The water dividing line is located at its western end. It is located near the Shaghib passageway to the south of the city of Abha (Salim, 1999, p. 101), Jabal al-Souda south of the city of Abha and the Its height is 3015 meters above sea level (Topographic map, 1:500000,1403 H) Proximity to him Mount Fares and between Abha and Souda rises Mount exultation, a lower rise and covered evergreen trees such as juniper. To the north of the hurdle of the motto, the mountains begin to rise and rough with the emergence of many distant peaks. These mountains are a mountain that is blocked near Tanumah, south of the city of Namas. It is considered the highest mountain in the western region of Asir and appears in the form of a plateau at an altitude of 2,950 meters above sea level (Salem, 2006, P. 108), map of ruggedness- index shown in Fig. (3).



Fig.(3):Map of ruggedness- index of study area. 


\section{-Degrees and slopes direction:}

The degree and direction of the slope of the area affects many variables and geomorphological forms, including the drainage network and hydrological variables, as well as the movement of materials on the slopes, which have a role on the geomorphological hazards in the region and the map of the slopes in the region. Mountain areas where the gradient reaches about $60 \square$. In general, the area descends to the north as shown in Fig. (4), which shows the trends of regression in the area. The study area is characterized by extreme gradations due to a number of valleys of high-rise to the heights in the western and southern regions, which is characterized by rugged Ptdharysha, as prevalent severe regressions western and southern parts, which represent the edge of Sarawat, and spread some severe slopes and represents more dangerous areas.



Fig. (4): The Degrees and direction of slopes of study area

\section{Geomorphologic Units}

The various geomorphic units and their component were identified and mapped. The descriptions of different geomorphic units of study area are given below, the study area can be divided into two main morphological units, each with distinct geomorphological characteristics fig. (5) 




Fig. (5): Geomorphological Units Map

\section{Mountains range:}

Occupies the southern and western section of the study area, In the form of unconnected mountain ranges, is a group of mountain clusters that extend almost straight, this mountainous mass separates from each other lateral and long valleys, which have played a major role in their extension and direction. The mountain range extends from 1500 meters to more than 3,000 meters. This elevation exceeds some of the high mountain peaks, such as the "Souda" mountain, 3015 meters above sea level, west of the of Abhaa urban, and near of it there is mountain of "Al-Faas". Between Abha and Souda there is a mountain of "Al-Ghara" at an altitude of 2700 meters above sea level (Salem, 2009, p. 214). This range includes the following geomorphological phenomena:

\section{i. Fault Scarps:}

Fault Scarps are the most important geomorphological forms associated with faults. They originate from rock movement and displacement due to the tension or pressure associated with the cracking process. They are very steep. They have evolved along a fault line due to the different weathering and erosion on both sides of the rift. This is reflected in the paths, directions and forms of valleys and their geomorphological characteristics. Several ridges have been identified in the southern and western regions of the study area in the mountains of "Al-Souda". The mountain ridges are characterized by their longitudinal lanes and small lanes in the north and north-west direction.

\section{ii. Hogback -Razorback:}

The slope of the gradient of more than 52 degrees is characterized by a strong gradient of overlapping layers of rock (cotton, 1950.p25). It is shown in the lithological weaknesses and has been observed in several sites in the study area, especially in the northern, north eastern and north western parts of the region studying.

\section{iii. Potholes:}

The three types of vascular rigs are located in the bottoms of most of the upper parts of the valleys of the study area. They are geomorphological phenomena that accompany 
valleys at the beginning of the geomorphological cycle due to the dominance of the vertical sculpting process and the deepening of the valleys. Potholes (Alexander, 1932, p56) are classified into three types: drilling of diving pools, drilling holes, shallow concave pits.

\section{iv. Gorges:}

The gorges are formed by the process of vertical erode and deepening of the valleys, especially if the valley passes through a cracking edge or follows a line fault (Torab, 2005, p183). The main valleys and others appear on the tributaries of the valleys.

\section{Plateaus and Piedmont range:}

This range is the northern and eastern parts of the study area. This range represents the transition zone between the mountain range, known as the Piedmont range or the range of the mountain feet. This range is the sedimentation surface of the valleys and the spherical fans consisting of gravel, glamide, rock masses and sand. The surface of the scale is characterized by the relative flat and gradient of the gradient from west to east. It is characterized by a strong separation due to tectonic movements.

\section{i. Floodplains}

The most important forms of geomorphology and the most important forms of sedimentation in the region. The surface sediments of the region consisted of gravel, limestone, rock masses and soft deposits of sand, mud and clay, with coarse sediments at the head of the plains, followed by medium sediments in the middle and soft deposits near the valleys. (Al-Koumi, 2004, p. 1455).

The main parameters of the sewage network and drainage basins, which amounted to about 46 water basins, were divided into three categories: morphometric variables, geomorphological variables, morphohydrological variables, and hydrologic variables. Table (2), (3) and Fig. (6) shows the most important morphological variables of the sewage system and basins in the study area, whether variables are included in the classification of basins according to their degree of variation or variables, are considered inputs for other variables See classification. 
Table (2) : Morphometric variables basins and streams of study area

\begin{tabular}{|c|c|c|c|c|c|c|c|c|c|c|c|c|c|c|}
\hline $\begin{array}{c}\text { No } \\
\dot{B a} \\
\text { sin }\end{array}$ & $\begin{array}{c}\mathrm{N} . \\
\text { stre } \\
\text { am }\end{array}$ & $\begin{array}{c}\text { T. } \\
\text { len } \\
\text { gth } \\
\text { of } \\
\text { se } \\
\text { we } \\
\mathbf{r} \\
\text { (k } \\
\text { m) }\end{array}$ & $\begin{array}{c}\begin{array}{c}\text { Strea } \\
\text { m } \\
\text { Frequ } \\
\text { ency } \\
) \\
\text { strea } \\
\text { m / } \\
(\mathbf{k m} 2\end{array}\end{array}$ & $\begin{array}{c}\text { Drai } \\
\text { nage } \\
\text { Dens } \\
\text { ity }\end{array}$ & $\begin{array}{l}\text { Slo } \\
\text { pes }\end{array}$ & $\begin{array}{c}\text { Consta } \\
\text { nt of } \\
\text { Chann } \\
\text { el } \\
\text { Mainte } \\
\text { nance }\end{array}$ & $\begin{array}{c}\text { Sinu } \\
\text { osity } \\
\text { Rati } \\
\text { o }\end{array}$ & $\begin{array}{c}\text { Bas } \\
\text { in } \\
\text { Ar } \\
\text { ea } \\
\mathbf{K} \\
\mathbf{M}^{2}\end{array}$ & $\begin{array}{l}\text { Bas } \\
\text { in } \\
\text { Len } \\
\text { gth } \\
\text { KM }\end{array}$ & $\begin{array}{c}\text { Bas } \\
\text { in } \\
\text { Wi } \\
\text { dth } \\
\text { K } \\
\text { M }\end{array}$ & $\begin{array}{c}\text { Wa } \\
\text { ter } \\
\text { Div } \\
\text { ide } \\
\text { Lin } \\
\text { e }\end{array}$ & $\begin{array}{l}\text { Circul } \\
\text { arity } \\
\text { Ratio }\end{array}$ & $\begin{array}{l}\text { elong } \\
\text { ation } \\
\text { ratio }\end{array}$ & $\begin{array}{c}\text { for } \\
\mathbf{m} \\
\text { fac } \\
\text { tor }\end{array}$ \\
\hline 1 & 139 & $\begin{array}{l}103 \\
.2\end{array}$ & 1.82 & 1.35 & $\begin{array}{c}2.4 \\
0\end{array}$ & 0.74 & 1.13 & $\begin{array}{l}76 . \\
31\end{array}$ & $\begin{array}{c}22.6 \\
1\end{array}$ & $\begin{array}{c}3.3 \\
8\end{array}$ & $\begin{array}{c}5.0 \\
0\end{array}$ & 38.34 & 0.22 & $\begin{array}{c}0.1 \\
5\end{array}$ \\
\hline 2 & 52 & $\begin{array}{l}44 . \\
0\end{array}$ & 1.73 & 1.46 & $\begin{array}{c}2.1 \\
8\end{array}$ & 0.68 & 1.13 & $\begin{array}{c}30 . \\
11\end{array}$ & $\begin{array}{c}33.2 \\
9\end{array}$ & $\begin{array}{c}0.9 \\
0\end{array}$ & $\begin{array}{l}35 . \\
07\end{array}$ & 0.31 & 0.09 & $\begin{array}{c}0.0 \\
3\end{array}$ \\
\hline 3 & 29 & $\begin{array}{l}18 . \\
7\end{array}$ & 2.18 & 1.41 & $\begin{array}{c}1.3 \\
5\end{array}$ & 0.71 & 1.12 & $\begin{array}{l}13 . \\
29\end{array}$ & 7.44 & $\begin{array}{c}1.7 \\
9\end{array}$ & $\begin{array}{r}23 . \\
69\end{array}$ & 0.30 & 0.28 & $\begin{array}{c}0.2 \\
4\end{array}$ \\
\hline 4 & 93 & $\begin{array}{l}81 . \\
3\end{array}$ & 1.57 & 1.37 & $\begin{array}{c}1.9 \\
0\end{array}$ & 0.73 & 1.12 & $\begin{array}{l}59 . \\
37\end{array}$ & $\begin{array}{c}19.6 \\
4\end{array}$ & $\begin{array}{c}3.0 \\
2\end{array}$ & $\begin{array}{c}60 . \\
10\end{array}$ & 0.21 & 0.22 & $\begin{array}{c}0.1 \\
5\end{array}$ \\
\hline 5 & 149 & $\begin{array}{l}152 \\
.2\end{array}$ & 1.43 & 1.46 & $\begin{array}{c}3.0 \\
8\end{array}$ & 0.68 & 1.15 & $\begin{array}{c}103 \\
.91\end{array}$ & $\begin{array}{c}22.9 \\
7\end{array}$ & $\begin{array}{c}4.5 \\
2\end{array}$ & $\begin{array}{l}70 . \\
88\end{array}$ & 0.26 & 0.25 & $\begin{array}{c}0.2 \\
0\end{array}$ \\
\hline 6 & 73 & $\begin{array}{l}56 . \\
1\end{array}$ & 1.69 & 1.30 & $\begin{array}{c}2.7 \\
6\end{array}$ & 0.77 & 1.11 & $\begin{array}{l}43 . \\
20\end{array}$ & $\begin{array}{c}35.1 \\
3\end{array}$ & $\begin{array}{c}1.2 \\
3\end{array}$ & $\begin{array}{c}52 . \\
61\end{array}$ & 0.20 & 0.11 & $\begin{array}{c}0.0 \\
4\end{array}$ \\
\hline 7 & 54 & $\begin{array}{l}51 . \\
7\end{array}$ & 1.46 & 1.40 & $\begin{array}{c}2.9 \\
2\end{array}$ & 0.71 & 1.12 & $\begin{array}{c}36 . \\
91\end{array}$ & $\begin{array}{c}15.9 \\
5\end{array}$ & $\begin{array}{c}2.3 \\
1\end{array}$ & $\begin{array}{l}47 . \\
37\end{array}$ & 0.21 & 0.21 & $\begin{array}{c}0.1 \\
5\end{array}$ \\
\hline 8 & 32 & $\begin{array}{l}28 . \\
2\end{array}$ & 1.75 & 1.54 & $\begin{array}{c}1.4 \\
9\end{array}$ & 0.65 & 1.14 & $\begin{array}{l}18 . \\
33\end{array}$ & 7.92 & $\begin{array}{c}2.3 \\
1\end{array}$ & $\begin{array}{c}26 . \\
24\end{array}$ & 0.33 & 0.31 & $\begin{array}{c}0.2 \\
9\end{array}$ \\
\hline 9 & 41 & $\begin{array}{l}24 . \\
7\end{array}$ & 2.30 & 1.38 & $\begin{array}{c}2.1 \\
8\end{array}$ & 0.72 & 1.12 & $\begin{array}{l}17 . \\
84\end{array}$ & 9.01 & $\begin{array}{c}1.9 \\
8\end{array}$ & $\begin{array}{l}29 . \\
35\end{array}$ & 0.26 & 0.26 & $\begin{array}{c}0.2 \\
2\end{array}$ \\
\hline 10 & 62 & $\begin{array}{l}44 . \\
7\end{array}$ & 1.94 & 1.40 & $\begin{array}{c}1.8 \\
8\end{array}$ & 0.71 & 1.12 & $\begin{array}{l}31 . \\
89\end{array}$ & $\begin{array}{c}10.7 \\
6\end{array}$ & $\begin{array}{c}2.9 \\
6\end{array}$ & $\begin{array}{c}34 . \\
59\end{array}$ & 0.33 & 0.30 & $\begin{array}{c}0.2 \\
8\end{array}$ \\
\hline 11 & 22 & $\begin{array}{l}27 . \\
0\end{array}$ & 1.26 & 1.54 & $\begin{array}{c}1.4 \\
9\end{array}$ & 0.65 & 1.11 & $\begin{array}{l}17 . \\
46\end{array}$ & $\begin{array}{c}41.9 \\
7\end{array}$ & $\begin{array}{c}0.4 \\
2\end{array}$ & $\begin{array}{r}47 . \\
01\end{array}$ & 0.10 & 0.06 & $\begin{array}{c}0.0 \\
1\end{array}$ \\
\hline 12 & 63 & $\begin{array}{l}60 . \\
8\end{array}$ & 1.64 & 1.58 & $\begin{array}{c}2.3 \\
4\end{array}$ & 0.63 & 1.13 & $\begin{array}{l}38 . \\
38\end{array}$ & $\begin{array}{c}18.6 \\
9\end{array}$ & $\begin{array}{c}2.0 \\
5\end{array}$ & $\begin{array}{c}54 . \\
92\end{array}$ & 0.16 & 0.19 & $\begin{array}{c}0.1 \\
1\end{array}$ \\
\hline 13 & 53 & $\begin{array}{l}73 . \\
3\end{array}$ & 1.17 & 1.62 & $\begin{array}{c}1.6 \\
8\end{array}$ & 0.62 & 1.15 & $\begin{array}{l}45 . \\
30\end{array}$ & $\begin{array}{c}20.3 \\
0\end{array}$ & $\begin{array}{c}2.2 \\
3\end{array}$ & $\begin{array}{l}53 . \\
34\end{array}$ & 0.20 & 0.19 & $\begin{array}{c}0.1 \\
1\end{array}$ \\
\hline 14 & 60 & $\begin{array}{l}34 . \\
8\end{array}$ & 2.34 & 1.36 & $\begin{array}{c}2.2 \\
0\end{array}$ & 0.74 & 1.10 & $\begin{array}{c}25 \\
65\end{array}$ & 8.80 & $\begin{array}{c}2.9 \\
2\end{array}$ & $\begin{array}{c}34 . \\
59\end{array}$ & 0.27 & 0.32 & $\begin{array}{c}0.3 \\
3\end{array}$ \\
\hline 15 & 45 & $\begin{array}{l}33 . \\
5\end{array}$ & 1.68 & 1.25 & $\begin{array}{c}2.0 \\
4\end{array}$ & 0.80 & 1.12 & $\begin{array}{l}26 . \\
84\end{array}$ & $\begin{array}{c}11.6 \\
6\end{array}$ & $\begin{array}{c}2.3 \\
0\end{array}$ & $\begin{array}{l}37 . \\
45\end{array}$ & 0.24 & 0.25 & $\begin{array}{c}0.2 \\
0\end{array}$ \\
\hline 16 & 52 & $\begin{array}{l}44 . \\
5 \\
\end{array}$ & 1.71 & 1.46 & $\begin{array}{c}4.4 \\
9 \\
\end{array}$ & 0.68 & 1.14 & $\begin{array}{l}30 . \\
46 \\
\end{array}$ & $\begin{array}{c}14.1 \\
7 \\
\end{array}$ & $\begin{array}{c}2.1 \\
5\end{array}$ & $\begin{array}{c}44 . \\
88 \\
\end{array}$ & 0.19 & 0.22 & $\begin{array}{c}0.1 \\
5 \\
\end{array}$ \\
\hline 17 & 80 & $\begin{array}{l}69 . \\
2\end{array}$ & 1.64 & 1.42 & $\begin{array}{c}2.6 \\
6\end{array}$ & 0.71 & 1.13 & $\begin{array}{l}48 . \\
87\end{array}$ & $\begin{array}{c}19.9 \\
9\end{array}$ & $\begin{array}{c}2.4 \\
5\end{array}$ & $\begin{array}{l}61 . \\
74\end{array}$ & 0.16 & 0.20 & $\begin{array}{c}0.1 \\
2\end{array}$ \\
\hline 18 & 67 & $\begin{array}{l}35 . \\
6\end{array}$ & 2.54 & 1.35 & $\begin{array}{c}3.5 \\
5\end{array}$ & 0.74 & 1.11 & $\begin{array}{l}26 . \\
33 \\
\end{array}$ & $\begin{array}{c}12.4 \\
6\end{array}$ & $\begin{array}{c}2.1 \\
1\end{array}$ & $\begin{array}{c}38 . \\
73\end{array}$ & 0.22 & 0.23 & $\begin{array}{c}0.1 \\
7 \\
\end{array}$ \\
\hline 19 & 46 & $\begin{array}{l}36 . \\
9\end{array}$ & 1.89 & 1.51 & $\begin{array}{c}2.2 \\
8\end{array}$ & 0.66 & 1.13 & $\begin{array}{l}24 . \\
37\end{array}$ & $\begin{array}{c}14.4 \\
1\end{array}$ & $\begin{array}{c}1.6 \\
9\end{array}$ & $\begin{array}{l}41 . \\
77\end{array}$ & 0.18 & 0.19 & $\begin{array}{c}0.1 \\
2\end{array}$ \\
\hline 20 & 232 & $\begin{array}{l}162 \\
.8\end{array}$ & 1.91 & 1.34 & $\begin{array}{c}4.9 \\
9\end{array}$ & 0.75 & 1.11 & $\begin{array}{l}121 \\
.54\end{array}$ & $\begin{array}{c}25.0 \\
1\end{array}$ & $\begin{array}{c}4.8 \\
6\end{array}$ & $\begin{array}{l}75 . \\
38\end{array}$ & 0.27 & 0.25 & $\begin{array}{c}0.1 \\
9\end{array}$ \\
\hline 21 & 124 & $\begin{array}{l}128 \\
.1 \\
\end{array}$ & 1.42 & 1.47 & $\begin{array}{c}1.7 \\
9 \\
\end{array}$ & 0.68 & 1.13 & $\begin{array}{l}87 . \\
06\end{array}$ & $\begin{array}{c}24.1 \\
5\end{array}$ & $\begin{array}{c}3.6 \\
0 \\
\end{array}$ & $\begin{array}{l}71 . \\
49 \\
\end{array}$ & 0.21 & 0.22 & $\begin{array}{c}0.1 \\
5 \\
\end{array}$ \\
\hline 22 & 75 & $\begin{array}{l}72 . \\
7\end{array}$ & 1.54 & 1.50 & $\begin{array}{c}1.7 \\
7\end{array}$ & 0.67 & 1.15 & $\begin{array}{l}48 . \\
57\end{array}$ & $\begin{array}{c}24.2 \\
2\end{array}$ & $\begin{array}{c}2.0 \\
1\end{array}$ & $\begin{array}{l}68 . \\
32\end{array}$ & 0.13 & 0.16 & $\begin{array}{c}0.0 \\
8\end{array}$ \\
\hline 23 & 302 & $\begin{array}{l}264 \\
.9\end{array}$ & 1.58 & 1.38 & $\begin{array}{c}3.7 \\
5\end{array}$ & 0.72 & 1.14 & $\begin{array}{l}191 \\
.50\end{array}$ & $\begin{array}{c}36.5 \\
6\end{array}$ & $\begin{array}{c}5.2 \\
4\end{array}$ & $\begin{array}{l}110 \\
.34\end{array}$ & 0.20 & 0.21 & $\begin{array}{c}0.1 \\
4\end{array}$ \\
\hline 24 & 81 & $\begin{array}{l}57 . \\
6 \\
\end{array}$ & 1.90 & 1.35 & $\begin{array}{c}2.0 \\
8 \\
\end{array}$ & 0.74 & 1.11 & $\begin{array}{l}42 . \\
56 \\
\end{array}$ & $\begin{array}{c}17.1 \\
4 \\
\end{array}$ & $\begin{array}{c}2.4 \\
8 \\
\end{array}$ & $\begin{array}{l}56 . \\
39\end{array}$ & 0.17 & 0.21 & $\begin{array}{c}0.1 \\
4 \\
\end{array}$ \\
\hline
\end{tabular}




\begin{tabular}{|c|c|c|c|c|c|c|c|c|c|c|c|c|c|c|}
\hline 25 & 117 & $\begin{array}{l}120 \\
.0\end{array}$ & 1.52 & 1.56 & $\begin{array}{c}1.6 \\
3\end{array}$ & 0.64 & 1.15 & $\begin{array}{l}77 . \\
00\end{array}$ & $\begin{array}{c}28.5 \\
3\end{array}$ & $\begin{array}{c}2.7 \\
0\end{array}$ & $\begin{array}{l}79 . \\
28\end{array}$ & 0.15 & 0.17 & $\begin{array}{c}0.0 \\
9\end{array}$ \\
\hline 26 & 51 & $\begin{array}{l}39 . \\
5\end{array}$ & 1.85 & 1.43 & $\begin{array}{c}1.8 \\
2\end{array}$ & 0.70 & 1.11 & $\begin{array}{l}27 . \\
64\end{array}$ & $\begin{array}{c}13.6 \\
0\end{array}$ & $\begin{array}{c}2.0 \\
3\end{array}$ & $\begin{array}{l}42 . \\
20\end{array}$ & 0.19 & 0.22 & $\begin{array}{c}0.1 \\
5\end{array}$ \\
\hline 27 & 361 & $\begin{array}{l}342 \\
.8\end{array}$ & 1.61 & 1.53 & $\begin{array}{c}2.2 \\
0\end{array}$ & 0.65 & 1.13 & $\begin{array}{c}224 \\
.00\end{array}$ & $\begin{array}{c}66.3 \\
8\end{array}$ & $\begin{array}{c}3.3 \\
7\end{array}$ & $\begin{array}{l}157 \\
.59\end{array}$ & 0.11 & 0.13 & $\begin{array}{c}0.0 \\
5\end{array}$ \\
\hline 28 & 178 & $\begin{array}{l}156 \\
.7 \\
\end{array}$ & 1.52 & 1.34 & $\begin{array}{c}5.0 \\
9 \\
\end{array}$ & 0.75 & 1.13 & $\begin{array}{r}116 \\
.94 \\
\end{array}$ & $\begin{array}{c}35.0 \\
5 \\
\end{array}$ & $\begin{array}{c}3.3 \\
4 \\
\end{array}$ & $\begin{array}{l}97 . \\
79 \\
\end{array}$ & 0.15 & 0.17 & $\begin{array}{c}0.1 \\
0 \\
\end{array}$ \\
\hline 29 & 43 & $\begin{array}{l}27 . \\
5 \\
\end{array}$ & 2.33 & 1.49 & $\begin{array}{c}1.9 \\
6 \\
\end{array}$ & 0.67 & 1.10 & $\begin{array}{l}18 . \\
45 \\
\end{array}$ & 7.28 & $\begin{array}{c}2.5 \\
3 \\
\end{array}$ & $\begin{array}{c}28 . \\
92\end{array}$ & 0.28 & 0.33 & $\begin{array}{c}0.3 \\
5 \\
\end{array}$ \\
\hline 30 & 84 & $\begin{array}{l}72 . \\
6\end{array}$ & 1.58 & 1.37 & $\begin{array}{c}2.5 \\
4\end{array}$ & 0.73 & 1.14 & $\begin{array}{c}53 . \\
14 \\
\end{array}$ & $\begin{array}{c}18.9 \\
7\end{array}$ & $\begin{array}{c}2.8 \\
0\end{array}$ & $\begin{array}{c}60 . \\
28\end{array}$ & 0.18 & 0.22 & $\begin{array}{c}0.1 \\
5\end{array}$ \\
\hline 31 & 110 & $\begin{array}{l}113 \\
.3\end{array}$ & 1.54 & 1.58 & $\begin{array}{c}2.4 \\
1\end{array}$ & 0.63 & 1.13 & $\begin{array}{l}71 . \\
52\end{array}$ & $\begin{array}{c}33.3 \\
7\end{array}$ & $\begin{array}{c}2.1 \\
4\end{array}$ & $\begin{array}{c}91 . \\
58\end{array}$ & 0.11 & 0.14 & $\begin{array}{c}0.0 \\
6\end{array}$ \\
\hline 32 & 103 & $\begin{array}{l}95 . \\
2\end{array}$ & 1.62 & 1.50 & $\begin{array}{c}1.9 \\
7\end{array}$ & 0.67 & 1.13 & $\begin{array}{c}63 . \\
59\end{array}$ & $\begin{array}{c}21.7 \\
3\end{array}$ & $\begin{array}{c}2.9 \\
3\end{array}$ & $\begin{array}{c}65 . \\
95\end{array}$ & 0.18 & 0.21 & $\begin{array}{c}0.1 \\
3\end{array}$ \\
\hline 33 & 37 & $\begin{array}{l}23 . \\
1\end{array}$ & 2.16 & 1.35 & $\begin{array}{c}1.6 \\
8\end{array}$ & 0.74 & 1.10 & $\begin{array}{l}17 . \\
15\end{array}$ & 8.83 & $\begin{array}{c}1.9 \\
4\end{array}$ & $\begin{array}{l}30 . \\
38\end{array}$ & 0.23 & 0.26 & $\begin{array}{c}0.2 \\
2\end{array}$ \\
\hline 34 & 144 & $\begin{array}{l}134 \\
.8 \\
\end{array}$ & 1.52 & 1.42 & $\begin{array}{c}2.0 \\
2 \\
\end{array}$ & 0.70 & 1.12 & $\begin{array}{l}94 . \\
92 \\
\end{array}$ & $\begin{array}{c}23.5 \\
8 \\
\end{array}$ & $\begin{array}{c}4.0 \\
3 \\
\end{array}$ & $\begin{array}{l}72 . \\
89 \\
\end{array}$ & 0.22 & 0.23 & $\begin{array}{c}0.1 \\
7 \\
\end{array}$ \\
\hline 35 & 125 & $\begin{array}{l}121 \\
.0\end{array}$ & 1.49 & 1.44 & $\begin{array}{c}3.4 \\
3\end{array}$ & 0.69 & 1.13 & $\begin{array}{l}83 . \\
99\end{array}$ & $\begin{array}{c}29.2 \\
2\end{array}$ & $\begin{array}{c}2.8 \\
7\end{array}$ & $\begin{array}{c}85 . \\
74\end{array}$ & 0.14 & 0.18 & $\begin{array}{c}0.1 \\
0\end{array}$ \\
\hline 36 & 273 & $\begin{array}{l}237 \\
.6 \\
\end{array}$ & 1.57 & 1.37 & $\begin{array}{c}4.1 \\
6 \\
\end{array}$ & 0.73 & 1.13 & $\begin{array}{l}173 \\
.41 \\
\end{array}$ & $\begin{array}{c}37.9 \\
5 \\
\end{array}$ & $\begin{array}{c}4.5 \\
7 \\
\end{array}$ & $\begin{array}{r}116 \\
.97 \\
\end{array}$ & 0.16 & 0.20 & $\begin{array}{c}0.1 \\
2 \\
\end{array}$ \\
\hline 37 & 843 & $\begin{array}{l}744 \\
.6\end{array}$ & 1.60 & 1.41 & $\begin{array}{c}2.4 \\
4\end{array}$ & 0.71 & 1.13 & $\begin{array}{r}527 \\
.51\end{array}$ & $\begin{array}{c}65.5 \\
9\end{array}$ & $\begin{array}{c}8.0 \\
4\end{array}$ & $\begin{array}{r}353 \\
.96\end{array}$ & 0.05 & 0.20 & $\begin{array}{c}0.1 \\
2\end{array}$ \\
\hline 38 & 38 & $\begin{array}{l}23 . \\
8\end{array}$ & 2.08 & 1.30 & $\begin{array}{c}3.1 \\
1 \\
\end{array}$ & 0.77 & 1.10 & $\begin{array}{l}18 . \\
30\end{array}$ & $\begin{array}{c}12.1 \\
2 \\
\end{array}$ & $\begin{array}{c}1.5 \\
1 \\
\end{array}$ & $\begin{array}{r}36 . \\
60 \\
\end{array}$ & 0.17 & 0.20 & $\begin{array}{c}0.1 \\
2 \\
\end{array}$ \\
\hline 39 & 161 & $\begin{array}{l}150 \\
.4\end{array}$ & 1.45 & 1.36 & $\begin{array}{c}2.3 \\
2\end{array}$ & 0.74 & 1.14 & $\begin{array}{c}110 \\
.99\end{array}$ & $\begin{array}{c}25.5 \\
7\end{array}$ & $\begin{array}{c}4.3 \\
4\end{array}$ & $\begin{array}{c}84 . \\
52\end{array}$ & 0.20 & 0.23 & $\begin{array}{c}0.1 \\
7\end{array}$ \\
\hline 40 & 90 & $\begin{array}{l}72 . \\
9\end{array}$ & 1.79 & 1.45 & $\begin{array}{c}2.0 \\
2\end{array}$ & 0.69 & 1.16 & $\begin{array}{c}50 . \\
16\end{array}$ & $\begin{array}{c}18.0 \\
0\end{array}$ & $\begin{array}{c}2.7 \\
9\end{array}$ & $\begin{array}{c}50 . \\
66\end{array}$ & 0.25 & 0.22 & $\begin{array}{c}0.1 \\
5\end{array}$ \\
\hline 41 & 126 & $\begin{array}{l}118 \\
.0\end{array}$ & 1.54 & 1.44 & $\begin{array}{c}2.1 \\
5 \\
\end{array}$ & 0.69 & 1.12 & $\begin{array}{l}81 . \\
84 \\
\end{array}$ & $\begin{array}{c}31.1 \\
2 \\
\end{array}$ & $\begin{array}{c}2.6 \\
3 \\
\end{array}$ & $\begin{array}{c}83 . \\
85 \\
\end{array}$ & 0.15 & 0.16 & $\begin{array}{c}0.0 \\
8 \\
\end{array}$ \\
\hline 42 & 55 & $\begin{array}{l}45 . \\
4\end{array}$ & 1.63 & 1.35 & $\begin{array}{c}4.0 \\
4 \\
\end{array}$ & 0.74 & 1.12 & $\begin{array}{c}33 . \\
71 \\
\end{array}$ & $\begin{array}{c}16.8 \\
4\end{array}$ & $\begin{array}{c}2.0 \\
0 \\
\end{array}$ & $\begin{array}{c}54 . \\
98 \\
\end{array}$ & 0.14 & 0.19 & $\begin{array}{c}0.1 \\
2 \\
\end{array}$ \\
\hline 43 & 421 & $\begin{array}{l}317 \\
.5 \\
\end{array}$ & 1.78 & 1.34 & $\begin{array}{c}3.0 \\
9 \\
\end{array}$ & 0.75 & 1.12 & $\begin{array}{c}236 \\
.93 \\
\end{array}$ & $\begin{array}{c}42.3 \\
8\end{array}$ & $\begin{array}{c}5.5 \\
9 \\
\end{array}$ & $\begin{array}{l}139 \\
.08 \\
\end{array}$ & 0.15 & 0.20 & $\begin{array}{c}0.1 \\
3 \\
\end{array}$ \\
\hline 44 & 190 & $\begin{array}{l}155 \\
.7 \\
\end{array}$ & 1.74 & 1.43 & $\begin{array}{c}3.1 \\
5 \\
\end{array}$ & 0.70 & 1.14 & $\begin{array}{l}109 \\
.05 \\
\end{array}$ & $\begin{array}{c}23.7 \\
1 \\
\end{array}$ & $\begin{array}{c}4.6 \\
0 \\
\end{array}$ & $\begin{array}{l}75 \\
26 \\
\end{array}$ & 0.24 & 0.25 & $\begin{array}{c}0.1 \\
9 \\
\end{array}$ \\
\hline 45 & 232 & $\begin{array}{l}190 \\
.6 \\
\end{array}$ & 1.64 & 1.35 & $\begin{array}{c}3.6 \\
4\end{array}$ & 0.74 & 1.14 & $\begin{array}{l}141 \\
.66 \\
\end{array}$ & $\begin{array}{c}34.8 \\
8\end{array}$ & $\begin{array}{c}4.0 \\
6\end{array}$ & $\begin{array}{l}105 \\
.16 \\
\end{array}$ & 0.16 & 0.19 & $\begin{array}{c}0.1 \\
2\end{array}$ \\
\hline 46 & 267 & $\begin{array}{l}103 \\
.2\end{array}$ & 1.82 & 1.35 & $\begin{array}{c}2.7 \\
7\end{array}$ & 0.74 & 1.13 & $\begin{array}{l}177 \\
.19\end{array}$ & $\begin{array}{c}22.6 \\
1\end{array}$ & $\begin{array}{c}3.3 \\
8\end{array}$ & $\begin{array}{c}5.0 \\
0\end{array}$ & 0.20 & 0.22 & $\begin{array}{c}0.1 \\
5\end{array}$ \\
\hline
\end{tabular}


Table (3): Determination of the depth and size of the surface runoff.

\begin{tabular}{|c|c|c|c|c|c|}
\hline No. Basin & $\mathbf{C N}$ & $\mathbf{S}$ & $\mathbf{L a}$ & $\begin{array}{c}\text { surface } \\
\text { runoff }(Q) \\
\text { at } M . \\
\text { Downfall } \\
(183.8 \text { mm }) \\
\end{array}$ & $\begin{array}{c}\text { Total flow } \\
\left(\mathbf{1 0 0 0 m}^{3}\right)\end{array}$ \\
\hline 1 & 92.29 & 21.22 & 4.24 & 160.58 & 12253.87 \\
\hline 2 & 91.65 & 23.13 & 4.63 & 158.68 & 4777.81 \\
\hline 3 & 91.00 & 25.12 & 5.02 & 156.75 & 2083.14 \\
\hline 4 & 92.10 & 21.80 & 4.36 & 160.00 & 9498.39 \\
\hline 5 & 88.04 & 34.49 & 6.90 & 148.04 & 15382.82 \\
\hline 6 & 92.49 & 20.63 & 4.13 & 161.17 & 6962.28 \\
\hline 7 & 93.04 & 19.01 & 3.80 & 162.81 & 6009.31 \\
\hline 8 & 91.00 & 25.12 & 5.02 & 156.75 & 2872.54 \\
\hline 9 & 91.42 & 23.84 & 4.77 & 157.99 & 2818.60 \\
\hline 10 & 92.24 & 21.38 & 4.28 & 160.42 & 5115.64 \\
\hline 11 & 91.00 & 25.12 & 5.02 & 156.75 & 2737.13 \\
\hline 12 & 92.95 & 19.26 & 3.85 & 162.55 & 6239.47 \\
\hline 13 & 91.00 & 25.12 & 5.02 & 156.75 & 7101.01 \\
\hline 14 & 90.92 & 25.35 & 5.07 & 156.52 & 4015.63 \\
\hline 15 & 90.95 & 25.27 & 5.05 & 156.61 & 4204.08 \\
\hline 16 & 94.54 & 14.67 & 2.93 & 167.30 & 5096.38 \\
\hline 17 & 92.63 & 20.21 & 4.04 & 161.59 & 7896.86 \\
\hline 18 & 92.36 & 21.00 & 4.20 & 160.80 & 4233.51 \\
\hline 19 & 94.93 & 13.56 & 2.71 & 168.47 & 4104.96 \\
\hline 20 & 89.23 & 30.67 & 6.13 & 151.51 & 18414.97 \\
\hline 21 & 92.20 & 21.50 & 4.30 & 160.30 & 13956.36 \\
\hline 22 & 91.36 & 24.02 & 4.80 & 157.82 & 7664.87 \\
\hline 23 & 88.46 & 33.13 & 6.63 & 149.26 & 28584.28 \\
\hline 24 & 90.14 & 27.79 & 5.56 & 154.20 & 6562.52 \\
\hline 25 & 91.49 & 23.64 & 4.73 & 158.19 & 12180.80 \\
\hline 26 & 89.12 & 31.00 & 6.20 & 151.21 & 4179.12 \\
\hline 27 & 91.80 & 22.69 & 4.54 & 159.12 & 35642.59 \\
\hline 28 & 90.71 & 26.01 & 5.20 & 155.90 & 18230.45 \\
\hline 29 & 90.45 & 26.81 & 5.36 & 155.13 & 2861.34 \\
\hline 30 & 91.66 & 23.10 & 4.62 & 158.72 & 8434.70 \\
\hline 31 & 92.09 & 21.82 & 4.36 & 159.98 & 11441.85 \\
\hline 32 & 92.95 & 19.26 & 3.85 & 162.55 & 10336.99 \\
\hline 33 & 91.70 & 23.00 & 4.60 & 158.82 & 2723.00 \\
\hline 34 & 90.35 & 27.13 & 5.43 & 154.82 & 14696.20 \\
\hline 35 & 91.59 & 23.31 & 4.66 & 158.51 & 13313.27 \\
\hline 36 & 89.63 & 29.39 & 5.88 & 152.70 & 26480.17 \\
\hline 37 & 91.06 & 24.93 & 4.99 & 156.93 & 82784.16 \\
\hline 38 & 92.84 & 19.59 & 3.92 & 162.22 & 2968.12 \\
\hline 39 & 87.54 & 36.15 & 7.23 & 146.56 & 16267.37 \\
\hline 40 & 91.14 & 24.71 & 4.94 & 157.15 & 7882.65 \\
\hline 41 & 89.87 & 28.64 & 5.73 & 153.40 & 12554.87 \\
\hline 42 & 90.99 & 25.16 & 5.03 & 156.71 & 5282.58 \\
\hline 43 & 93.07 & 18.92 & 3.78 & 162.90 & 38595.75 \\
\hline 44 & 90.94 & 25.29 & 5.06 & 156.58 & 17074.86 \\
\hline
\end{tabular}






Fig(6) : Basins and stream order of study area.

\section{ii. Rocky hills}

One advantage of integrating the scanned symbol based geomorphological map into GIS is that it forms a background image and thereby remains a valuable source of information in interpreting geomorphological hazards. For example, the variability of material cover and process intensity, expressed by changing the density of symbols is still recognizable and increases the practical value of the various GIS layers. This means that the source of information upon which the decisions are made is visible to others. A further advantage is that the final maps do not rely only on instability features alone but are based on a full representation of the landscape. A further strength of GIS is also that both quantitative and qualitative terrain parameters can easily be integrated.

The hills are characterized by their igneous and metamorphic formation, and vary in forms of structures, some conical and some domes, whether alone or contiguous, and classified as follows:

\section{i. Rock outcrops. \\ ii. volcanic cones. \\ iii. Isenberg. \\ iv.}

\section{Urban Expansion}

It is evident from the interpretation and integration of multi-temporal and multiresolution aerospace data and other maps that Abah area has experienced a significant urban growth in the period of 1980 to 2018. The change detection shows that the urban area of Abah was respectively $28 \mathrm{~km}^{2}$ in 1980, $56 \mathrm{~km}^{2}$ in 1985, $129 \mathrm{~km}^{2}$ in $1990,204 \mathrm{~km}^{2}$ in 1995 , $215 \mathrm{~km}^{2}$ in $2000,255 \mathrm{~km}^{2}$ in $2005,302 \mathrm{~km}^{2}$ in $2010,380 \mathrm{~km}^{2}$ in 2015 , and $410 \mathrm{~km}^{2}$ in 2018 (fig.7). It's had extended by $\mathrm{km}$ in the period 1980-2018 at a growth rate of $50 \mathrm{~km}^{2} / 5 \mathrm{year}$. The computed total area of Abah metropolitan raban (including Abha town; Khamis Mushayt, Ahad Rufaydah, Alfareayn, Tandiha, Alwadiin, Alshaeaf, Tabab; and AS sudaho). 




Fig.(7): Urban Expansion Map of the Study Area. 


\section{5. geomorphic hazards}

The study area includes main geomorphic hazards the following:

\section{i. Seismic hazard}

The risk of damage from earthquakes is quite low over most of Saudi Arabia, the main areas of risk being near the Gulf of Aqaba and Jizan, with lower risk in the west near the Red Sea. Abha urban is a mountainous zonation, was accompanied by large geodynamic movements linked to active faults, is growing concern on the volcanic activity associated with earthquakes in the Red Sea have been the work of affluent studies to assess the seismic risk level. Western Region of Saudi Arabia is considered to be a moderately active seismic zone these induce natural hazards are not without effect on the lives of people and the whole area fig. (8) Distribution of earthquakes recorded in the period $1974-2018$, Distribution of earthquakes according to the map and their frequency according to the Richter scale (162 earthquakes).

According to this information this area saves 3 to 4 earthquakes per year, fig. (9). Earthquakes have never caused any casualties in area. But, in the case of major human concentrations at the study area. that is the most obvious aspect of the exposure of people to risks. It is also possible that some mass movements have a close relationship with earthquakes, the largest earthquake suffered by the region, with a magnitude of four degrees on the Richter scale. at 4:06:52 local time, On the morning of Friday, 14/2 / 1439H (3/11/2017), the quake is located northwest of the city of Nemas a distance of $16 \mathrm{~km}$ (Saudi Geological Survey,2018)



Fig.(8):Distribution of earthquakes recorded in the period $1974-2018$. 




Fig.(9):The intensity of occurrence of earthquakes

\section{Flash floods hazards}

Flooding is regarded as one of the most dangerous natural hazards and as a principal trigger of disasters (Alc'antara-Ayala, 2002). One of the methods to curb the catastrophic effects of floods is to use hazard maps applied to regional/local planning management with the cooperation of national and local authorities (Montz and Gruntfest, 2002).

Flash floods is intended to quantities of rain, which is more than the absorbance capacity of the soil or its permeability in the basin area, leading to the flow of water in the form of multi-directional random paths, according to the topography and slope of the basin surfaces, which are accumulated in sub-streams, to pour into the main drains. to study the flow Spate Identification of risks, and its destructive ability, Flood risk is the combination of two factors, hazard and vulnerability. The flood hazard defines itself as vulnerability determines the land and its use. Thus, the consequences of this hazard will be less important in uninhabited and urban areas. Flood hazards are perceived better in urban than rural areas. It's the case of floods. Its occurrence was related to heavy rainfall that hit the mountainous part of the city, drained. The causes are related to the anarchic occupation in prohibited areas by building and also deforestation in mountainous areas $(1500-3000 \mathrm{~m})$ devoted to green spaces. the flood is a valley of Dalih, which happened in $1402 \mathrm{AH}, 1982 \mathrm{AD}$, one of the most destructive and violent floods, water flow reached speeds of more than 13 meters / second. the flood has destroyed the road obstacle of Dalih Comprehensive destruction. and it was rebuilt and inaugurated (Mahsoub, 2009, p.79).

\section{1- The amount of runoff Spate:}

It has been estimated depth of runoff, depending on model (SCS- CN), The following figures illustrate the types of land cover that have been relied upon to classify soils Fig. (10$\mathrm{A})$, and to determine the values of $\mathrm{CN}$ So land cover fig. (10-B), And then calculate the net depth of annual surface flow (mm) fig.(11- A), Annual flow volume $\left(1000 \mathrm{~mm}^{3}\right)$ fig.(11-B), through the analysis of Abha climatic station, and estimate the annual rainfall rate of about $(183.8 \mathrm{~mm} /$ year) table.(4), and to rely on this rate as an input in the net flow calculation equations $(\mathrm{Q})$, annual flow volume $\left(\mathrm{m}^{3}\right)$, Which introduces the most important criteria for classification of the degree of risk of basins in the study area. 
Table.(4): The Maximum of rainfall in Abha station (MM).

\begin{tabular}{|l|c|c|c|c|c|c|c|c|c|c|c|r|}
\hline station & JAN & FEB & MAR & APR & MAY & JUN & JUL & AUG & SEP & OCT & NOV & \multicolumn{2}{|l|}{} \\
\hline $\begin{array}{l}\text { Maximum } \\
\text { of rainfall }\end{array}$ & $\mathbf{8 0 . 6}$ & $\mathbf{3 6 2 . 0}$ & $\mathbf{4 1 5 . 0}$ & $\mathbf{2 4 4 . 0}$ & $\mathbf{1 1 2 . 8}$ & $\mathbf{5 8 . 3}$ & $\mathbf{8 9 . 7}$ & $\mathbf{1 1 3 . 9}$ & $\mathbf{4 4 . 2}$ & $\mathbf{2 4 . 0}$ & $\mathbf{4 3 . 7}$ & 29.6 \\
\hline Year & 1992 & $\mathbf{1 9 8 3}$ & 1997 & $\mathbf{2 0 1 6}$ & $\mathbf{2 0 1 3}$ & $\mathbf{1 9 9 6}$ & $\mathbf{1 9 7 8}$ & $\mathbf{1 9 9 2}$ & $\mathbf{2 0 1 1}$ & $\mathbf{2 0 0 1}$ & $\mathbf{2 0 1 3}$ & 1989 \\
\hline
\end{tabular}

Source: General Authority for Meteorology, during the period from 1978 to 2017.



Fig.(10): The Curve numbers values CNS For land cover(A), average curved numbers for drainage basins.



Fig. (11): Surface flow of basins(A), average numbers of curved basins main drainage and $\operatorname{streams}(B)$. 


\section{2- Grade of riskiness}

An average rick of the of the basins of the valleys in the Abha urban is about 2.95 degree (scale 1: 5), the highest risk is about 3.33 degree, and whereas less serious about 1.77 degree fig. (12)



Fig.(12):Flash floods hazards in the study area.

\section{ii. mass movements hazards}

originally associated with mountainous regions can occur even in the lowlands. They are usually caused by a combination of three factors including geology (regolith water soaked, discontinuity, schistocyte, dikes, joints and faults contrast in permeability and rigidity of the material, earthquake), geomorphological (tectonics and subsidence, erosion and groundwater extraction, etc..) and human (deforestation, agricultural activities, mining, urbanization, slopes excavation etc. ).

The general cause of the movement of materials on the slopes in increasing the driving forces and the Reduces the resistance forces (Merrits, D.J., et al., 1998, p. 184), That is, what controls the degree of stability of rock blocks and make them more stable several factors, the most important characteristics rock, and the systems of joints and cracks effect of weathering, and the degree of regression and the shape of the slope, the layers has be tend in a direction South-East at an angle ranging from 40 to 50 degree, and the extent of saturation of water sediments, the extent of plant coverings and rarity it, and the quantity and size of compresses, in addition to human activity and removal of plants(Maatouq,2001,pp.663665).the movement of materials on the slopes of several operations, including rockfall and is a quick and sudden operations, When the blocks break up, they fall down through the air through the free fall, and then after colliding with the ground moving rolling and being broken. it is the risk on the roads and construction located below the edge of the mountains as well as the flash flood in study area, so, it's study in depend on model of the mass movements hazards fig.(13), by standards taken into consideration to draw the map of Landslide 
vulnerability in study area and its relative weights table.(5), in addition to drawing map of the classification of slopes according to Yong fig.(14), so as to draw a map of Landslide vulnerability zones of the study area fig.(15).



Table.(5): standards taken into consideration to draw the map of Landslide vulnerability in study area.

\begin{tabular}{|c|c|}
\hline Relative weight & Standards \\
\hline 0.3 & Ruggedness Index \\
\hline 0.3 & Geological formation \\
\hline 0.25 & The slopes \\
\hline 0.15 & faults and dikes \\
\hline $\mathbf{1}$ & Total weights \\
\hline
\end{tabular}






Fig.(14):Map of the classification of slopes according to Yong of the study area.



Fig.(15): Map of Landslide vulnerability zones of the study area. 


\section{Conclusions and recommendations:}

Geomorphological hazards like flash floods and mass movements are destructive and frequently occurring phenomena all over the world. The study area has been affected by flash floods and mass deposition hazards frequently. These hazards strike different types of infrastructures as well as urban areas in the study area causing property damages. These geomorphological hazards acting on the study area will be accelerated due to many reasons. One of them is due to the lack of proper warning information about flash floods and mass depositions hazards for the people pass through and live in the prone areas. Another reason is due to severe lack of land use planning. This might result in even more damages in near future. The evaluation of the geomorphological hazards was carried out on the basis of analyzing the flash flood and mass deposition in the study area, also to analyze an area with different susceptibility to geomorphological hazards. The current research exploits the utilization of by GIS techniques, combined with remote sensing data, topographic maps and field data for geomorphological hazard analysis in the study areas. Integration between geological, geomorphological, climate, remote sensing, urban, and infrastructure distribution have been used to evaluate the geomorphological hazards. As a conclusion, the hazard and susceptibility maps proved to be very useful tools for determine the hazard areas as well as the most susceptible zones that need mitigation measures and advanced planning. Although flooding and mass deposition are natural phenomena which cannot be completely avoided, however by establishing good mitigation strategies, the disastrous effects can be reduced marginally. Furthermore, a GIS database can help the decision makers to make decisions at time of need. so, it is recommended to improve the present situation and control the future expansion of the Study area.

To mitigate the geomorphological hazard level, it is necessary to determine the most susceptible hazard zones and above all Fig (16), to reduce the mass wasting problems and falsh flood hazards. Generally, mitigation measures should be taken for flood and mass deposition protection Fig (16). It complements other preventive tools like the effective planning of the growth of cities by creating a computerized GIS database for the flood-prone and mass deposition-prone areas. For the flash flood hazards in the study area, set of barriers are proposed at locations in the upstream parts of each basin as well as in the down slope's areas. The high damaging effect of the flash flood is mostly caused by the transportation of big boulders. In that case, the barriers will prevent the boulders to move and the proposed dams will help in saving the runoff water. In addition, a warning system has to be established to alarm people before the floods. On the other hand, for the mass depositional problems on expansion urban different suggestions are made as follows:

- The proposed dams to obstruct and slowdown the floodwater current and its load or to spill it through culverts and store it behind the dams.

- To increase the slope shoulder width to avoid the rocks falling from reaching the roads.

- To create a ditch under natural slopes and rock cuts that will take up all fallen material.

- For the critical zones to install a wire mesh that covers (part of) the slopes to decrease the energy of the fallen rocks.

- To put warning signs about the areas most affected by mass deposits and fallen rocks.

- Leaving space for the valleys, as a limit for the expansion of urban areas at a distance of not less than $50 \mathrm{M}$, it can be increased or reduced depending on the situation.

- The Preferably when protection from the dangers of floods, use of the area roc ,especially granite and basalt rocks, where they have the ability to resist weathering factors.

- among the most used techniques for the mobilization and the good management of the water, one quotes. the example of the mechanical benches, the cords in dry stones the 
individual cuvette as well as the hill lakes, In addition to prickly pear. It must be taken into consideration.



Fig(16): Map of the most susceptible hazard zones and stability of the study area.

\section{Sources and references:}

\section{First: Sources.}

1- Saudi Geological Survey, National Centre for Earthquake and Volcanoes. Tectonics of the Arabian Plate, 2018. Available at: http://www.sgs.org.sa/English/NaturalHazards/Pages/Earthquakes.aspx.

\section{Second: Arabic References:}

1. Abdullah Allam Abdo Allam (1999): Geomorphological Hazards on the Mountain Roads in Southwest Saudi Arabia - A Study in Applied Geomorphology, Journal of the Faculty of Arts, Menoufia University, Issue Fourteen.

2. Ahmed El Sayed Maatouq (2001) Geomorphological features of landslides in rib valley: (Case study, Abha), Journal of Imam Muhammad bin Saud Islamic University, no. 32, pp. 651-699.

3. Tariq Zakaria Ibrahim Salem (2009) Climate and Tourism in Abha Urban Area, Saudi Arabia, Applied Climate Study, Journal of the Faculty of Arts, Zagazig University, pp. 252-206.

4. Mohamed Sabri Mahsoub (1998): Natural hazards and disasters, a study in physical geography, Dar Elfakir ELaribe, Cairo.

\section{Third: English References.}

1- Alc'antara-Ayala, I. (2002): Geomorphology, natural hazards, vulnerability and prevention of natural disasters in developing countries, Geomorphology, 47, 107-124. 
2- A. M. Youssef, B. Pradhan, A. F. D. Gaber, and M. F. Buchroithner(2009): Geomorphological hazard analysis along the Egyptian Red Sea coast between Safaga and Quseir, Natural Hazards and Earth System Sciences, V., 9, pp.751-766.

3- By William ( 1983)Geologic map of the ABHA Quadrangle, Sheet 18 F,Kingdom of Saudi Arbia.

4- Cooke, R.U. (1976): Urban geomorphology. Geographical Journal. Volume 142. pp. 59-65.

5- Cooke, R.U., Brunsden, D., Doornkamp, J.C., and Jones, D.K.C., (1982): Urban geomorphology in Drylands. Oxford University Press, Oxford, pp. 324.

6- C. Fern andez-Lavado, G. Furdada, and M. A. Marqu'es(2007): Geomorphological method in the elaboration of hazard maps for flash-floods in the municipality of Jucuar'an (El Salvador), Natural Hazards and Earth System Sciences, V., 7,pp. 455465.

7- HORTON, R.E., (1945): Drainage basin characteristic, geophysics. Union. Trans., V., 13, PP. 350-361.

8- Crichton,D.(1999): The Risk Triangle, edited by: Ingleton, J., Natural Disaster Management, Tudor Rose, London, 102-103.

9- Lazzari, M., Geraldi, E., Lapenna, V., and Loperte, A. (2006): Natural hazards vs. human impact: an integrated methodological approach in geomorphological risk assessment on the Tursi historical site, Southern Italy, Landslides, 3, 275-287.

10-Miller, V., (1953): Aquantitative Geomorphic Study of Drainage Basin Characteristics in the Clinch Mountain Area, project NR 389 - 042, Tech. Rept.3., Columbia uni.

11- Montz, B. E. and Gruntfest, E.: Flash-flood mitigation (2002): recommendations for research and applications, Environmental Hazards, 4, 15-22

12- Merrits, D.J., Andrew de Wet and Menking, K., (1998): Environment Geology: An Earth Science Approach, second edition., W.H, Freaman and Company, New York.

13- West, W.D., and Choubey, V.D., (1964): The Geomorphology of the Country around Sagar and Katangi (M.P.). Journal Geological Society of India. Vol. 5. pp. 41-55. 
خريطة الأخطار الجيومورفولوجية بأبها الحضرية، المملكة العربية السعودية .

$$
\text { د/ منا العسال }
$$

الملخص:

تقع أبها الحضرية في منطقة عسير، جنوب غرب المملكة العربية السعودية. تعد الجيومورفولوجيا واحدة من أكثر الضوابط المؤثرة في التخطيط الحضري، والتي تعطي فكرة عن الاختلافات في المظاهر



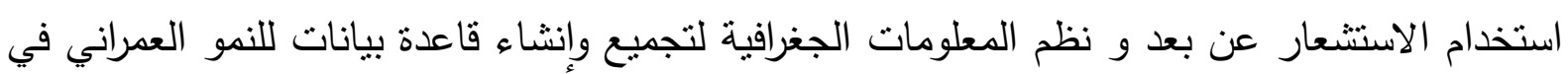

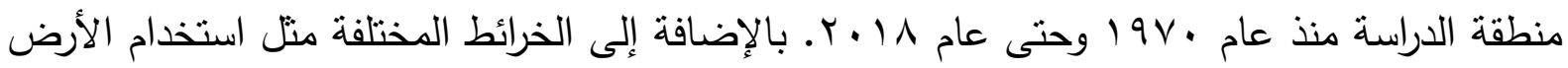
والخرائط الطبوغرافية لتحليل الارتفاع والتضاريس. والجيومورفولوجيا تسلط الضوء على على التغييرات في المظاهر الطبيعية والأشكال الأرضية التي تطورت بشكل تدريجي مع مرور الوقت. من خلال دراسة ولفية




المعلومات الجغرافية والاستشعار عن بعد لنقييم الاخطار الجيومورفولوجية. يعد تقييم الأخطار الجيولوجية أحد المكونات الهامة لتقييم الأخطار الطبيعية. وتعرض هذه الورقة الخرائط


البيانات الجيولوجية والجيومورفولوجية والبيانات الأخرى من نظم المعلومات الجغرافية، لإنتاج خرائط




الخطر على المناطق الحضرية، يمكن لهذه الخرائط أن تساعد في بدء إجراءات مناسبة للتخفيف من لإنى





الكلمات المفتاحية: الجيومورفولوجيا الحضرية، الأخطار الجيومورفولوجية، نظم المعلومات الجغرافية ، الاستشعار عن بعد. 